\title{
PREVALENCIA DEL SÍNDROME DE BURNOUT EN ESTUDIANTES DE MEDICINA HUMANA DE UNA UNIVERSIDAD PÚBLICA EN PERÚ.
}

\section{PREVALENCE OF BURNOUT SYNDROME IN HUMAN MEDICINE STUDENTS OF A PUBLIC UNIVERSITY OF ICA, PERU -2016}

\section{ARTÍCULO ORIGINAL}

Núnez-Joseli Luis Alfredo ${ }^{1, a}$, Bedriñana Curitomay Indira ${ }^{1, b}$, Mendoza Llamoca Carlos ${ }^{1, b}$, Zelada Rios Laura ${ }^{1, b}$

1. Facultad de Medicina Humana, Universidad Nacional San Luis Gonzaga. Ica, Perú

a. Médico Psiquiatra

b. Egresado

\section{Correspondencia:}

Mendoza Llamoca Carlos Miguel Dirección: Calle los Pacaes A-30, Urb. San José - Ica, Perú.

Teléfono: (51) 994777318

Correo Electrónico:

carlos_mll17@hotmail.com

Contribuciones De Autoría: MAII, GAAD, LMGB, CMB participaron en el diseño del estudio, el análisis de los datos, revisaron críticamente el artículo y aprobaron la versión final

Conflicto De Intereses: No declarados.

Financiamiento: Autofinanciado.

Como Citar

Núñez-Joseli L., Bedriñana I., Mendoza-Llamoca C., Zelada L. Prevalencia del síndrome de Burnout en estudiantes de medicina humana de una universidad pública de Ica, Perú- 2016. Rev méd panacea. 2017;6 (2): $60-63$

Recibido: 10-05-2017 Aceptado: 15-06-2017 Publicado: 20-06-2017

\begin{abstract}
RESUMEN:
Objetivo: Determinar la prevalencia del síndrome de Burnout y sus dimensiones, así como sus factores asociados en estudiantes de medicina de la Facultad de Medicina Humana "Daniel Alcides Carrión" de la Universidad Nacional San Luis Gonzaga de Ica. Materiales y Métodos: Se realizó un estudio transversal con los estudiantes de medicina pertenecientes al semestre académico 2016-II de la UNSLG. Se emplearon dos encuestas autoadministradas anónimas, una relacionada a las características sociodemográficas y otra, la escala de Maslach Burnout Inventory en su versión para estudiantes (MBI-SS) validada en español. Resultados: Se encuestó un total de 368 (59,5\%) estudiantes, donde la media de edad fue de 22.11, con una predominancia del sexo femenino con un $53 \%(n=195)$. La prevalencia del SBO fue del $12 \%(n=45)$, un $34,5 \%$ ( $n=127)$ mostraron niveles altos de agotamiento emocional, un 25\% ( $n=92)$ niveles altos de despersonalización, y un $28,8 \%(n=106)$ niveles bajos de realización personal. Conclusiones: Se encontró una baja prevalencia del SBO, aunque se muestra dentro de la tendencia revelada por otros estudios similares. La dimensión de agotamiento emocional fue la más prevalente, lo cual encaja dentro de la cascada de síntomas que desencadena finalmente el SBO.
\end{abstract}

Palabras Claves: Burnout, estudiantes, prevalencia

\section{ABSTRACT:}

Objetive: To establish the prevalence of burnout and its dimensions, as well as the associated factors in medical students of medicine school "Daniel Alcides Carrión" of the San Luis Gonzaga National University of Ica Materials and Methods: A transversal study was performed with medical students from UNSLG during academic semester 2016-II. Two anonymous self-administered questionnaires were assessed, one of them included sociodemographic characteristics and the other included the Maslach Burnout Inventory - Student Survey (MBI-SS) version validated in spanish. Results: A total of 368 (59,5\%) students were surveyed, where the mean age was 22.11 , with a predominance of females with $53 \%(n=195)$. The prevalence of SBO was $12 \%$ ( $n$ $=45), 34,5 \%(n=127)$ showed high levels of emotional exhaustion, $25 \%(n=92)$ high levels of depersonalization, and $28,8 \%=106$ ) low levels of personal fulfillment. Conclusions: A low prevalence of SBO was found, although it is shown within the trend revealed by other similar studies. The dimension of emotional exhaustion was the most prevalent, which fits within the cascade of symptoms that ultimately triggers SBO.

Keywords: Burnout, students, prevalence. 


\section{INTRODUCCIÓN}

Diversos estudios demuestran que las condiciones de trabajo de los médicos pueden ser extenuantes, física y emocionalmente, con una importante frecuencia de síndrome de Burnout, depresión y suicidio. Los estudiantes de medicina, en nuestro país, son considerados parte de los equipos de salud, en ellos la alta presión académica, la sobrecarga laboral, la inestabilidad financiera, la falta de sueño y la cercanía con el paciente, tienen un efecto negativo inadvertido, encontrándose en este grupo mayor prevalencia de psicopatología, depresión, ansiedad, abuso de sustancias e ideación suicida. $(1,2)$

Santander et al., en un estudio realizado en Chile reportó que la prevalencia de síntomas de trastornos mentales entre los estudiantes de medicina es mayor que lo encontrado en la población general. (3) Eróstegui et al en Bolivia, encontró un total de $27 \%$ de prevalencia de síndrome de Burnout en estudiantes de medicina. (4) También en nuestro país se han realizado diversos estudios en estudiantes de medicina, siendo la población objetivo en la mayoría de estos, los internos de medicina, por ejemplo Cordiva Huancas en su tesis realizada en internos de medicina de la Universidad Ricardo Palma encontró una prevalencia de SBO de hasta 60,1\%(5).

El síndrome de agotamiento profesional, cuyo término en inglés es síndrome de Burnout (SBO), fue definido por primera vez por el psicólogo clínico Herbert J. Freudenberger en 1974 como un conjunto de síntomas inespecíficos médico-biológicos y psicosociales, que se desarrollan en la actividad laboral, como resultado de una demanda excesiva de energía, refiriéndose a los llamados profesionales de "ayuda" (6). Fue Christina Maslach (1976) quién convirtió al SBO en un verdadero producto de investigación. Si bien comenzó a estudiar el SBO como un "estado", en 1981 junto con Susan Jackson redefinen este síndrome, considerándolo ya no como un fenómeno estático resultante de un problema individual, intrapsíquico sino como un "proceso" mucho más complejo, y como una respuesta al estrés laboral crónico. Un síndrome que se caracteriza por tener tres dimensiones (7):

- $\quad$ Agotamiento emocional: Cansancio y fatiga que puede manifestarse física y/o psíquicamente, es la sensación descrita como no poder dar más de sí mismo a los demás.

- $\quad$ Despersonalización: Desarrollo de sentimientos, actitudes y respuestas negativas, distantes y frías hacia otras personas especialmente hacia los beneficiarios del propio trabajo.

- $\quad$ Falta de realización personal: Sentimiento de incompetencia que aparece cuando el sujeto siente que las demandas laborales exceden su capacidad, se encuentra insatisfecho con sus logros profesionales.

Dada la importancia del problema, el presente estudio busca establecer la prevalencia del síndrome de Burnout en estudiantes de medicina, observar las diferencias que se podrían presentar entre ellos de acuerdo a características relevantes asociadas a la aparición de esta entidad, con el fin de permitir el desarrollo de programas educativos orientados a la formación integral de los estudiantes, buscando una adecuada salud mental.

\section{MATERIAL Y MÉTODOS}

Se realizó un estudio transversal, descriptivo y observacional en una población constituida por la totalidad de estudiantes matriculados oficialmente en el I - XII ciclo del semestre académico 2016 II de la Facultad de Medicina "Daniel Alcides Carrión" de la UNSLG, siendo estos un total de 619 , El muestreo fue no probabilístico por conveniencia, para un tamaño muestral mínimo de 176 estudiantes, siendo calculado con la fórmula respectiva, con un nivel de confianza del 95\%; quedando finalmente la muestra constituida por 368 alumnos (59,5\%).

Para la recolección de información se recurrió a la técnica de la encuesta, empleándose un instrumento de recolección de datos que constó de dos partes: Datos generales, en el cual se recopilaron datos concernientes a características sociodemográficas de los estudiantes que participaron en el estudio, además de preguntas que buscan identificar factores que son relacionados frecuentemente con el SBO y el Cuestionario de MBI- SS que consta de 15 ítems que evalúan las tres dimensiones del SB: agotamiento emocional (5 ítems), despersonalización ( 4 ítems) y falta de realización personal (6 ítems) las cuales se valoraron con una escala tipo Likert de siete puntos, siendo o nunca y 6 todos los días.

Las calificaciones de cada dimensión se consideraron "bajo", "medio" o "alto". Se calificó como "alto" si se encontraban en el tercio superior de la distribución, "medio" si lo hacían en el tercio medio y "bajo" si se encontraban en el tercio inferior. Para definir la presencia del síndrome de Burnout se utilizó el criterio definido por Maslach caracterizado por altas puntuaciones en la dimensión de agotamiento emocional y despersonalización y bajas en realización personal.

El procesamiento de datos se realizó utilizando el programa estadístico SPSS versión 19, en el cual se realizó la estadística descriptiva y se relacionaron las variables, aplicando la prueba de chi cuadrado para establecer la significancia de dicha relación.

\section{RESULTADOS}

En este estudio se encuestaron a un total de 405 estudiantes de un total 619 alumnos matriculados en el semestre académico 2016-II, de dichas encuestas se consideraron 368 (59,5\%), excluyéndose así 37 encuestas por encontrarse ilegibles o incompletas. La media de edad del total de encuestas válidas fue de 22.11, además la distribución por sexo reveló un predominio del sexo femenino con un 53\% ( $n=195)$ frente al masculino con un $47 \%(n=173)$.

En cuanto a la prevalencia del SBO, se encontró $12 \%(n=45)$ según como se ha descrito en la metodología de cómo sería evaluado y diagnosticado este síndrome. En relación a las dimensiones del Síndrome de Burnout, se encontró un 34,5\% ( $n=127)$ de alumnos con niveles altos de Agotamiento emocional, un $25 \%(n=92)$ de alumnos con niveles altos de despersonalización, Finalmente en el caso de la Realización personal, se encontró un total de $28,8 \% \quad(n=106)$ de estudiantes con niveles bajos.

A cerca de la relación entre el Ciclo de estudios y la prevalencia de $\mathrm{SBO}$, encontramos la mayor prevalencia en el $\mathrm{V}$ y III ciclo, con $33,3 \%$ y $32,4 \%$ respectivamente. En contraparte, la menor prevalencia de SBO, la encontramos en VII y IX ciclo, con porcentaje nulo de afectados, siendo el nivel de significancia de estos parámetros menor de 0.05 con lo cual resulta significativo. (Tabla $\mathrm{N}^{\circ} 1$ )

\section{Tabla 1.}

Relación entre Ciclo de estudios y prevalencia de SBO en estudiantes de medicina de la Universidad Nacional San Luis Gonzaga de Ica, 2016

\begin{tabular}{|c|c|c|c|c|}
\hline & & NO SBO & SBO & Total \\
\hline \multirow{7}{*}{ CICLO DE } & \multirow{3}{*}{ II } & 88 & 4 & 92 \\
\hline & & $95,7 \%$ & $4,3 \%$ & $100 \%$ \\
\hline & & $23,9 \%$ & $1,1 \%$ & $25,0 \%$ \\
\hline & \multirow{3}{*}{ III } & 23 & 11 & 34 \\
\hline & & $67,6 \%$ & $32,4 \%$ & $100 \%$ \\
\hline & & $6,3 \%$ & $3 \%$ & $9,2 \%$ \\
\hline & \multirow{3}{*}{ IV } & 37 & 4 & 41 \\
\hline \multirow[t]{5}{*}{ ESTUDIOS } & & $90,2 \%$ & $9,8 \%$ & $100 \%$ \\
\hline & & $10,1 \%$ & $1,1 \%$ & $11,1 \%$ \\
\hline & \multirow{3}{*}{ V } & 16 & 8 & 24 \\
\hline & & $66,7 \%$ & $33,3 \%$ & $100 \%$ \\
\hline & & $4,3 \%$ & $2,2 \%$ & $6,5 \%$ \\
\hline
\end{tabular}




\begin{tabular}{|c|c|c|c|c|}
\hline & & 24 & 4 & 28 \\
\hline \multirow{20}{*}{$\begin{array}{l}\text { CICLO DE } \\
\text { ESTUDIOS }\end{array}$} & \multirow[t]{3}{*}{ VI } & $85,7 \%$ & $14,3 \%$ & $100 \%$ \\
\hline & & $6,5 \%$ & $1,1 \%$ & $7,6 \%$ \\
\hline & & 18 & 0 & 18 \\
\hline & \multirow[t]{3}{*}{ VII } & $100,0 \%$ & $0 \%$ & $100 \%$ \\
\hline & & $4,9 \%$ & $0 \%$ & $4,9 \%$ \\
\hline & & 24 & 8 & 32 \\
\hline & \multirow[t]{2}{*}{ VII } & $75 \%$ & $25,0 \%$ & $100 \%$ \\
\hline & & $6,5 \%$ & $2,2 \%$ & $8,7 \%$ \\
\hline & \multirow{4}{*}{ IX } & 22 & 0 & 22 \\
\hline & & $100 \%$ & $0 \%$ & $100 \%$ \\
\hline & & $6 \%$ & $0 \%$ & $6 \%$ \\
\hline & & 26 & 4 & 30 \\
\hline & \multirow[t]{3}{*}{$X$} & $86,7 \%$ & $13,3 \%$ & $100 \%$ \\
\hline & & $7,1 \%$ & $1,1 \%$ & $8,2 \%$ \\
\hline & & 31 & 1 & 32 \\
\hline & \multirow[t]{3}{*}{ XI } & $96,9 \%$ & $3,1 \%$ & $100 \%$ \\
\hline & & $8,4 \%$ & $0,3 \%$ & $8,7 \%$ \\
\hline & & 14 & 1 & 15 \\
\hline & \multirow[t]{2}{*}{ XII } & $93,3 \%$ & $6,7 \%$ & $100 \%$ \\
\hline & & $3,8 \%$ & $0,3 \%$ & $4,1 \%$ \\
\hline \multirow{2}{*}{\multicolumn{2}{|c|}{ Total }} & 323 & 45 & 368 \\
\hline & & $87,8 \%$ & $12,2 \%$ & 1 \\
\hline
\end{tabular}

Con respecto a la relación entre sexo y SBO (Gráfico $N^{\circ} 1$ ) se observa que es discretamente mayor en el caso del sexo masculino con $6,3 \%$ frente al $6 \%$ del femenino, $(p=0.5)$.

A cerca de los factores asociados al SBO, solo se encontró relación significativa con la actividad laboral (Gráfico $\mathrm{N}^{\circ} 2$ ) siendo que la prevalencia de este síndrome fue mayor entre aquellos que si laboraban constituyendo el $26,1 \%$ frente al $11,3 \%$ de los que se dedicaban exclusivamente a sus estudios $(p=0.03)$. Otros factores estudiados sin relación significativa fueron el estado civil, encontrando que del total de encuestados $98,1 \%$ eran solteros, de los cuales el porcentaje de SBO era de $11,9 \%,(p=0.447)$, la presencia de hijos, el cual, en nuestro estudio solo el $2,2 \%(n=8)$ de estudiantes tenían hijos $(p=0.981)$. Por otro lado en relación al tipo de vivienda de los estudiantes se encontró un predominio de estudiantes que vivían con familiares directos (padres y hermanos) siendo en total un $52,4 \%$ $(n=193)(p=0.239)$.

En relación a las horas de sueño, hubo un predominio de los estudiantes con 4-8 horas de sueño, siendo estos un $89,7 \%(n=252)$, $(\mathrm{p}=0.088)$. Tampoco se encontró relación significativa con la frecuencia de realización de actividades recreativas, consumo de sustancias como alcohol, drogas o tabaco, lugar de procedencia y tipo de familia.

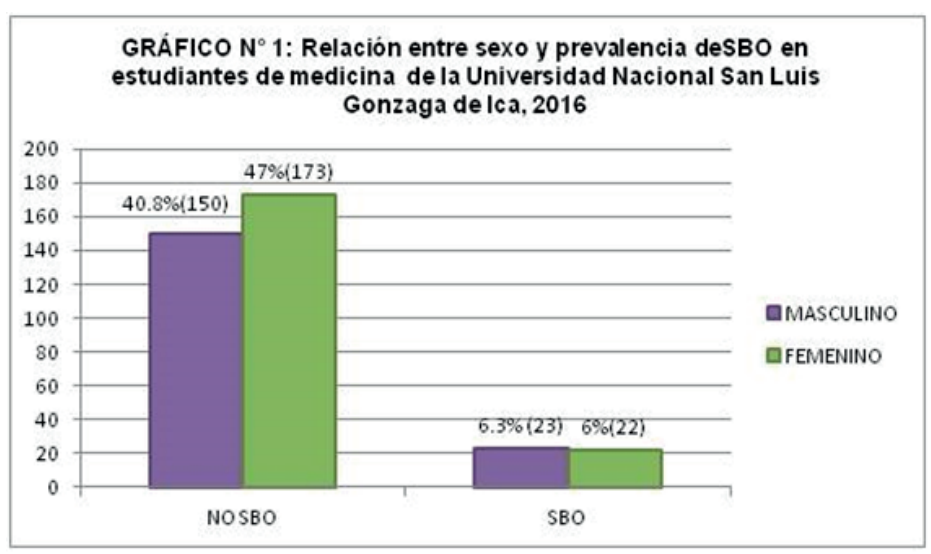

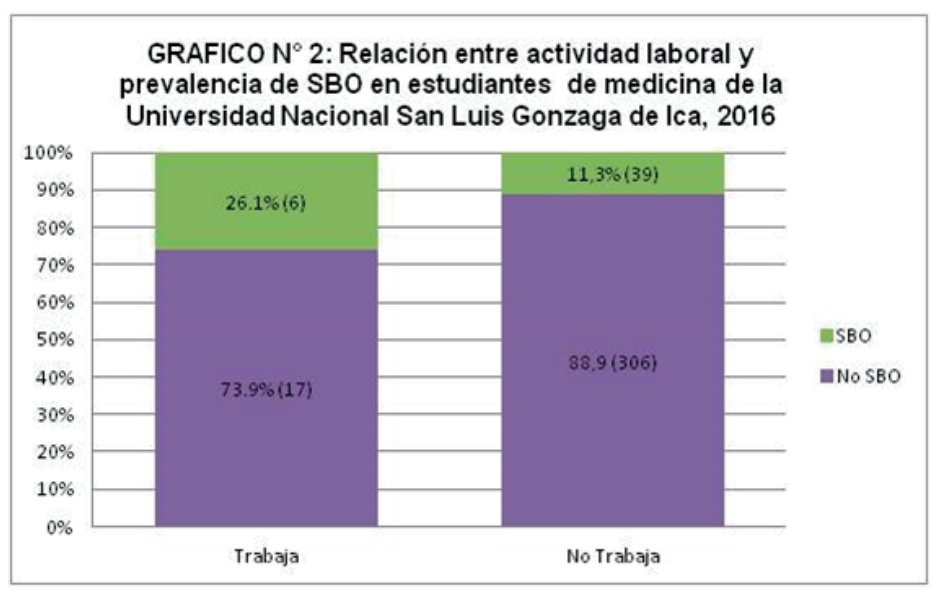

\section{DISCUSION}

En nuestro estudio se utilizaron los criterios de Maslach y Jackson (7) obteniendo en total un $12 \%$ de prevalencia de SBO, el cual es similar a los resultados obtenidos por Fontes de Oliva et al (8); de Mejía et al (9), con prevalencias que no superan el $10 \%$ en general.

La dimensión con mayor prevalencia fue la de Agotamiento emocional en niveles altos con un valor de $34,5 \%$, Realización personal con $28,8 \%$ en nivel bajo, y finalmente Despersonalización en nivel alto con 25\%; no coincidiendo con los estudios de Alfaro et al (10), en Chile, con valores de 63,3\%, para despersonalización 48,8\% para agotamiento emocional y $21,3 \%$ para baja realización personal y de Serrano et al (11), en Colombia, con $37,8 \%, 36,2 \%$ y $25,6 \%$, en agotamiento emocional, despersonalización y baja realización personal respectivamente.

Estudios manifiestan que este síndrome presenta una tendencia a su incremento conforme el estudiante se encuentra en un ciclo o año superior, ya sea en su prevalencia o en el grado de SBO que presenten $(11,12)$ aludiendo que podría deberse al mayor contacto con el paciente, sus dolencias y demás, pudiendo influir negativamente en el alumno.

En nuestro estudio, no se evidencia esta tendencia, ya que se observa que el ciclo con mayor proporción de SBO es el $\mathrm{V}$ y III ciclo, con 33,3\% y $32,4 \%$ de estudiantes de cada ciclo con SBO, pese a encontrarse ambos ciclos en las áreas pre-clínicas son los que presentan mayor prevalencia de SBO, podría atribuirse a que en las materias cursadas en dichos ciclos el estilo de enseñanza, forma de calificación o grado de dificultad de las mismas requiera un mayor esfuerzo por parte de los estudiantes generando respuestas de estrés, agotamiento, conllevando así al SBO. Estas distribuciones sin una tendencia definida evidente se observan también en estudios como el de Alfaro-Toloza et al (10) y Mejía et al (9) en Chile y Perú respectivamente.

Encontramos en este estudio que la prevalencia de SBO fue muy similar en ambos sexos, con una leve preponderancia en el masculino (6.3\% frente a 6\%). Existe discrepancia en este punto, así tenemos que si bien el estudio de Fontes de Oliva (8) muestra una mayor prevalencia en el sexo masculino (13,4\% contra 7,1\%), diversos estudios como el presentado por Backović(13) muestran una frecuencia de SBO mayor en el sexo femenino, encontrándose diferencias de sexo en la autopercepción de la salud física y el estrés que ocasionan determinadas actividades académicas, siendo las mujeres quienes presentan una peor respuesta a estos estresores académicos, declarando incluso como estresante el contacto con los pacientes.

Si bien la prevalencia de SBO fue baja, existe un gran porcentaje de estudiantes con alguno de las dimensiones que lo conforman alterados, principalmente el agotamiento emocional, que es el punto de apertura al tridente que constituye el SBO. Por lo que sería recomendable que la institución establezca medidas de prevención que aborden en forma eficiente el desarrollo conjunto del SBO en todas sus dimensiones, actuando asimismo en los distintos niveles de prevención: primario, secundario y terciario. 


\section{BIBLIOGRAFÍA}

1. Ramírez A, Medeiro M, Muñoz C, Ramírez G. Alcances del Síndrome de Burnout en estudiantes de medicina. Revista ANACEM. 2012; 6(2):110-112.

2. Estela M, Jiménez R, Landeo J, Tomateo J, Vega J. Prevalencia de síndrome de Burnout en alumnos del séptimo año de medicina de una universidad privada de Lima, Perú. Rev Neuropsiquiatr 2010; 73 (4): 147-156.

3. Santander J, Romero M, Hitschfeld M, Zamora V. Prevalencia de ansiedad y depresión entre los estudiantes de medicina de la Pontificia Universidad Católica de Chile. REV CHIL NEURO-PSIQUIAT 2011; 49 (1): 47-55.

4. Erostegui C, Gutierrez O, Espada G. Síndrome de burn-out en estudiantes de la Facultad de medicina de la UMSS junio-julio 2009. Rev. Méd.-Cient. “Luz Vida”. 2010; 1 (1): 5-8.

5. Cordiva C. Prevalencia del síndrome de Burnout en internos de medicina de la Facultad de Medicina Humana de la Universidad Ricardo Palma -2015. Tesis de pregrado. Universidad Ricardo Palma 2016.

6. Giberti E. Alerta y Cuidado de la salud de los operadores ante los efectos traumáticos de atención a las víctimas. Burnout. Revista de Derecho Penal Integrado. 2001; 2(3).
7. Maslach C; Jackson S. The measurement of experienced Burnout. Journal of Occupational Behaviour. 1981; 2:99-113.

8. Fontes de Oliva E, Andrade S, Rodrigues de Abreu A, Vieira E, Matos T. Burnout Syndrome and associated factors among medical students: a cross-sectional stundy. Clinics 2012; 67(6): 573-80.

9. Mejia R, Valladares J, Talledo L, Sánchez K, Rojas C, Ruiz J, et al. Síndrome de Burnout y factores asociados en estudiantes de medicina. Estudio multicéntrico en siete facultades de medicina peruanas. Rev Chil Neuro-Psiquiat 2016; 54 (3): 207-214.

10. Alfaro $P$, Olmos R, Fuentealba M, Céspedes-González E. Síndrome de Burnout y factores asociados en estudiantes de una escuela de medicina de Chile. CIMEL. 2013; 18(2):23-26.

11. Serrano F, Salguero J , Ayala J , García M , Meza J, Mejía C. Síndrome de Burnout en estudiantes de seis facultades de medicina de Colombia, 2016-1: estudio multicéntrico. CIMEL 2016; 21(2) 29-34. 12. Asencio L, Almaraz G, Huerta P, Silva L, Muñoz M, Monroy F. Síndrome de burnout en estudiantes de primero a sexto año de medicina en una universidad privada del norte de México: estudio descriptivo transversal. Medwave 2016;16(3):e6432.

13. Backović D, Živojinović J, Maksimović J, Maksimović M. Gender differences in academic stress and burnout among medical students in final years of education. Psychiatria Danubina, 2012; Vol. 24, No. 2, pp 175-181. 